\title{
Anomalous Dimension in a Two-Species Reaction-Diffusion System
}

\author{
Benjamin Vollmayr-Lee ${ }^{1}$, Jack Hanson ${ }^{2}$, R. Scott McIsaac ${ }^{3}$, and Joshua D. Hellerick ${ }^{1}$ \\ ${ }^{1}$ Department of Physics 85 Astronomy, Bucknell University, Lewisburg, PA 17837, USA \\ ${ }^{2}$ Department of Mathematics, City College of New York, \\ 160 Convent Ave, New York, NY 10031 USA and \\ ${ }^{3}$ Calico Life Sciences, South San Francisco, CA 94080 USA
}

\begin{abstract}
We study a two-species reaction-diffusion system with the reactions $A+A \rightarrow(0, A)$ and $A+B \rightarrow A$, with general diffusion constants $D_{A}$ and $D_{B}$. Previous studies showed that for dimensions $d \leq 2$ the $B$ particle density decays with a nontrivial, universal exponent that includes an anomalous dimension resulting from field renormalization. We demonstrate via renormalization group methods that the scaled $B$ particle correlation function has a distinct anomalous dimension resulting in the asymptotic scaling $\tilde{C}_{B B}(r, t) \sim t^{\phi} f(r / \sqrt{t})$, where the exponent $\phi$ results from the renormalization of the square of the field associated with the $B$ particles. We compute this exponent to first order in $\epsilon=2-d$, a calculation that involves 61 Feynman diagrams, and also determine the logarithmic corrections at the upper critical dimension $d=2$. Finally, we determine the exponent $\phi$ numerically utilizing a mapping to a four-walker problem for the special case of $A$ particle coalescence in one spatial dimension.
\end{abstract}

\section{INTRODUCTION}

Reaction-diffusion systems are known to exhibit a strong dependence on fluctuations in lower dimensions that renders mean-field rate equations invalid [1, 2]. For example, the $A+A \rightarrow 0$ annihilation reaction has rate equation $\partial_{t}\langle a\rangle=-\Gamma\langle a\rangle^{2}$, which provides a density decaying as $\langle a\rangle \sim 1 /(\Gamma t)$ with the nonuniversal rate constant $\Gamma$. But for dimensions $d \leq d_{c}=2$ nontrivial correlations develop and give rise to universal power-law behavior [3], the density decaying as $\langle a\rangle \sim A(D t)^{-d / 2}$ (with logarithmic corrections in $d=2$ ), where $D$ is the diffusion constant and $A$ is a universal amplitude [4]. Field-theoretic renormalization group (RG) methods have proved useful in analyzing this fluctuationdominated regime. See Ref. [5] for a review.

For many irreversible reactions, such as the annihilation reaction above, the resulting dynamical exponents (but not the amplitudes) may simply be determined from Smoluchowski theory [6, 7], which is an improved rate equation with a time-dependent reaction rate. The success of the Smoluchowski theory, in spite of being an uncontrolled approximation, stems from the lack of field renormalization in these theories, and thus the absence of an anomalous dimension. Counterexamples are processes with competing branching reactions, such as branchingannihilating random walks [8, 9] or directed percolation [10 12], for which field renormalization is required and nontrivial scaling exponents result.

Nevertheless, nontrivial exponents can arise in reaction-diffusion systems without branching reactions. We consider such a model here: two particle species, $A$ and $B$, with diffusion constants $D_{A}$ and $D_{B}$ undergo the reactions

$$
\begin{aligned}
& A+A \rightarrow \begin{cases}A & \text { (coalescence) prob. } p \\
0 & \text { (annihilation) prob. } 1-p .\end{cases} \\
& A+B \rightarrow A \quad \text { (trapping) }
\end{aligned}
$$

The rate equations, valid for $d>2$, are

$$
\partial_{t}\langle a\rangle=-\Gamma\langle a\rangle^{2} \quad \partial_{t}\langle b\rangle=-\Gamma^{\prime}\langle a\rangle\langle b\rangle,
$$

where the angle brackets represent averages with respect to the stochastic processes of diffusion and reaction, as well as over initial conditions. These result in $\langle a\rangle \sim 1 /(\Gamma t)$ and $\langle b\rangle \sim t^{-\theta}$ with the exponent $\theta=\Gamma^{\prime} / \Gamma$ given by nonuniversal rate constants. The fluctuation dominated case of $d \leq 2$ has been studied by the Smoluchowski approach [13] and by RG techniques [14 16 (with $A$ particle dynamics reducing to the well-studied single-species reaction [3, 4].) In contrast to the rate equation result, $\theta$ is found to be universal, depending only on the parameters $\delta=D_{B} / D_{A}$ and $p$. Smoluchowski theory gives

$$
\theta_{S}=\frac{d}{2-p}\left(\frac{1+\delta}{2}\right)^{d / 2}
$$

while the RG predicts

$$
\theta=\theta_{S}+\frac{1}{2} \gamma_{b}^{*}
$$

where the anomalous dimension $\gamma_{b}^{*}$, given in Eq. (34), is of order $\epsilon=2-d$ and stems from the necessary field renormalization of the $b$ density.

In the present work we demonstrate the existence of an additional anomalous dimension $\phi$ for this system which emerges from the scaled $B$ particle correlation function

$$
\tilde{C}_{B B}(r, t) \equiv \frac{\langle b(r, t) b(0, t)\rangle-\langle b(t)\rangle^{2}}{\langle b(t)\rangle^{2}} \sim t^{\phi} f(r / \sqrt{t})
$$

In contrast, the scaled correlation functions $\tilde{C}_{A A}$ and $\tilde{C}_{A B}$ are simply functions of $r / \sqrt{t}$ with no time dependent prefactor. This exponent results from the multiplicative renormalization factor $Z_{b^{2}}$ required by the $b^{2}$ density operator, causing $\left\langle b^{2}\right\rangle /\langle b\rangle^{2}$ to be renormalized 
by a factor $Z_{b^{2}} /\left(Z_{b}\right)^{2}$, while $\langle a b\rangle /(\langle a\rangle\langle b\rangle)$ has no corresponding factor. The case of $\left\langle a^{2}\right\rangle /\langle a\rangle^{2}$ is more subtle: as shown in [17], a multiplicative renormalization factor $Z_{a^{2}}$ is required due to the anticorrelation of the $A$ particles, but with the consequence that $\tilde{C}_{A A} \propto r / \sqrt{t}$ rather than anomalous time dependence of the form (5).

Smoluchowski theory gives $\phi_{S}=0$ due to the lack of field renormalization. We turn to the field-theoretic RG treatment to obtain a systematic expansion in powers of $\epsilon=2-d$, where the number of loops in the Feynman diagram expansion is equivalent to the resulting order of $\epsilon$. The tree level (zero loop) RG calculation also gives $\phi=0+O(\epsilon)$. At one-loop order the calculation involves 61 diagrams, giving

$$
\phi=\frac{13}{24-18 p} \epsilon+O\left(\epsilon^{2}\right),
$$

which is the primary result of this paper. The calculation demonstrates that the exponent is universal, depending only on $p$ and the diffusion constant ratio $\delta$, though curiously the $\delta$ dependence drops out to first order in $\epsilon$. Since $\phi$ is positive, the $B$ density fluctuations increase with time.

As a consequence of the anomalous dimension, the amplitudes of both $\langle b(t)\rangle$ and $\tilde{C}_{B B}(0, t)$ are nonuniversal for $d<2$. Asymptotically, these quantities are functions of the universal lengths $\sqrt{D_{A} t}$ and $\sqrt{D_{B} t}$ as well as a nonuniversal length scale $\Lambda$ that reflects the lattice spacing or capture radius, reaction rates, and other microscopic details. From dimensional analysis, $\langle b(t)\rangle \sim$ $t^{-d / 2} g(\Lambda / \sqrt{t}) \sim \Lambda^{2 \theta-d} t^{-\theta}$, and similarly, $\tilde{C}_{B B}(0, t)=$ $g(\Lambda / \sqrt{t}) \sim \Lambda^{-2 \phi} t^{\phi}$.

At the upper critical dimension $d_{c}=2$ we obtain the density

$$
\langle b\rangle \sim(\ln t)^{\alpha} t^{-(1+\delta) /(2-p)}
$$

where

$$
\alpha=\frac{3}{2}\left(\frac{1+\delta}{2-p}\right)+\frac{1}{2}\left(\frac{1+\delta}{2-p}\right)^{2} f(\delta)
$$

with $f(\delta)$ given in Eq. (30). The density decay exponent is necessarily discontinuous at the upper critical dimension $d_{c}=2$, since it is universal below and nonuniversal above $d_{c}$. The power law in (7) corresponds to the $\epsilon \rightarrow 0$ limit of (4), as was found by [14] and [16]; however, our exponent $\alpha$ differs from those previous works. We return to this point in the summary. We find the scaled correlations at the upper critical dimension to have the form

$$
\tilde{C}_{B B}(r, t) \sim(\ln t)^{\alpha_{2}} f(r / \sqrt{t})
$$

with

$$
\alpha_{2}=\frac{1+9 p}{12-9 p}
$$

For the case where the $A$ particles undergo the coalescence reaction $(p=1)$ in one spatial dimension, the exponent $\phi$ can be related to a four walker problem which we call the bracket problem: given four random walkers that begin spaced along a line, the probability that the middle two walkers ( $B$ 's) have not met either of the end walkers ( $A$ 's) by time $t$ decays as $t^{-\beta}$. We measure this exponent numerically with use of a mapping to an electrostatic problem [18, 19], and find for the case of equal diffusion constants that $\beta=1.873754(3)$. Through scaling arguments presented below, we have (for $p=\delta=d=1$ ) $\phi=5 / 2-\beta$, giving

$$
\phi \simeq 0.626246(3)
$$

In comparison, the $\epsilon$ expansion truncated at first order and evaluated at $\epsilon=1$ provides $\phi=13 / 6 \simeq 2.17$. Evidently the $\epsilon$ expansion is not rapidly convergent.

The layout of the paper is as follows. In Sec. II we introduce the field theory and diagrammatic expansion. In Sec. III we discuss the renormalization of the theory and review how it applies to the $B$ density. This is followed in Sec. IV by a calculation of correlation function anomalous dimension $\phi$ to order $\epsilon$, with details of this calculation provided in an appendix. The density and correlations at the upper critical dimension $d=2$ is discussed in Sec.V In Sec. VI we present the numerical calculation of $\phi$ that exploits a connection to the four-walker bracket problem. Finally, we summarize our results in Sec. [VII]

\section{FIELD THEORY}

The two-species model described in Eq. (11) is first written in terms of a probability master equation, and then following standard methods [5, 20 22] converted via Fock space to a field theory. The resulting action is

$$
\begin{aligned}
S=\int d^{d} x d & \left\{\bar{a}\left(\partial_{t}-\nabla^{2}\right) a+\bar{b}\left(\partial_{t}-\delta \nabla^{2}\right) b\right. \\
+ & \lambda \bar{a} a^{2}+\lambda \bar{a}^{2} a^{2}+\lambda^{\prime} Q \bar{b} a b+\lambda^{\prime} \bar{a} \bar{b} a b \\
+ & \left.\left(\bar{a} a_{0}+\bar{b} b_{0}\right) \delta(t)\right\} .
\end{aligned}
$$

Here $a$ and $b$ are complex fields corresponding to $A$ and $B$ particles, and $\bar{a}$ and $\bar{b}$ are auxiliary fields. The first line in (12) represents the diffusion process, with time rescaled so that $D_{A}=1$ (recall $\left.\delta=D_{B} / D_{A}\right)$. The second line represents the reaction processes with microscopic rate constants $\lambda$ for annihilation and coalescence and $\lambda^{\prime}$ for the trapping reaction. For notational simplicity we have introduced the parameter $Q=1 /(2-p)$. In the conventional mapping, a factor $1 / Q$ appears in the $\bar{a} a^{2}$ coefficient, reflecting the average number of particles removed by an $A+A$ reaction. In the action above we have rescaled $a \rightarrow Q a, \bar{a} \rightarrow \bar{a} / Q$ for convenience. The third line corresponds to Poissonian initial conditions with average densities $a_{0}$ (after rescaling) and $b_{0}$. The compo- 


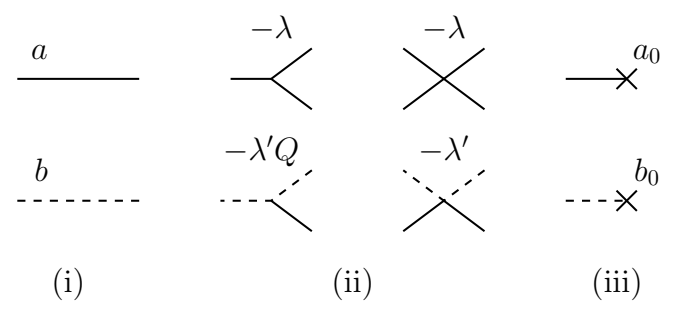

FIG. 1: Diagram ingredients: (i) the diffusion propagators, (ii) the interaction vertices, and (iii) the initial terms of the theory. Time flows from right to left.

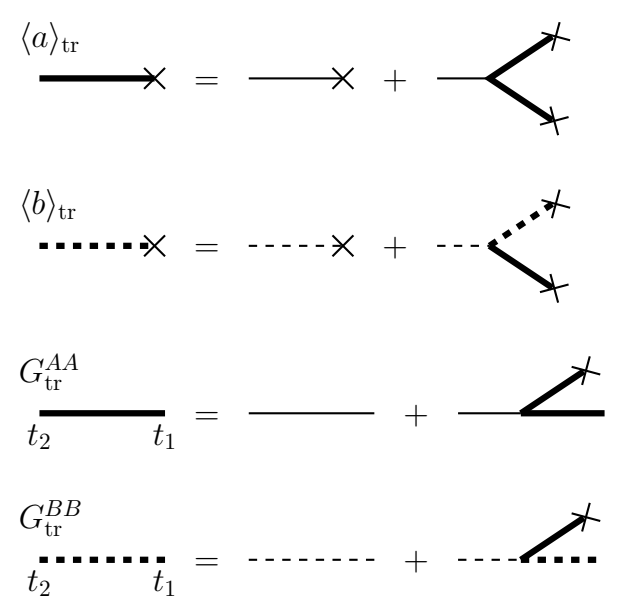

FIG. 2: Diagrammatic representation of the Dyson equations for the tree level densities and dressed propagators, which are depicted as bold lines.

nents of Feynman diagrams resulting from this action are shown in Fig. 1.

The averages of the $a$ and $b$ fields can be directly related to the densities and correlation functions of the $A$ and $B$ particles. These can be computed as an expansion in the number of loops, which results after renormalization in an $\epsilon=2-d$ expansion. An infinite number of diagrams result for each order in the loop expansion. To evaluate these infinite sums we need the tree-level (zero loop) densities $\langle a\rangle_{\text {tr }}$ and $\langle b\rangle_{\text {tr }}$ and dressed propagators $G_{\mathrm{tr}}^{A A}\left(\mathbf{k}, t_{2}, t_{1}\right)=\left\langle a\left(\mathbf{k}, t_{2}\right) \bar{a}\left(-\mathbf{k}, t_{1}\right)\right\rangle_{\mathrm{tr}}$ and $G_{\mathrm{tr}}^{B B}\left(\mathbf{k}, t_{2}, t_{1}\right)=\left\langle b\left(\mathbf{k}, t_{2}\right) \bar{b}\left(-\mathbf{k}, t_{1}\right)\right\rangle_{\mathrm{tr}}$. These can be computed from Dyson equations [4, 5, 16], shown in Fig. 2. resulting in

$$
\begin{aligned}
\langle a(t)\rangle_{\mathrm{tr}} & =\frac{a_{0}}{1+\lambda a_{0} t} \\
\langle b(t)\rangle_{\mathrm{tr}} & =\frac{b_{0}}{\left(1+\lambda a_{0} t\right)^{Q \lambda^{\prime} / \lambda}}
\end{aligned}
$$

and for $t_{2}>t_{1}$

$$
\begin{aligned}
& G_{\mathrm{tr}}^{A A}\left(\mathbf{k}, t_{2}, t_{1}\right)=\left(\frac{1+a_{0} \lambda t_{1}}{1+a_{0} \lambda t_{2}}\right)^{2} e^{-k^{2}\left(t_{2}-t_{1}\right)} \\
& G_{\mathrm{tr}}^{B B}\left(\mathbf{k}, t_{2}, t_{1}\right)=\left(\frac{1+a_{0} \lambda t_{1}}{1+a_{0} \lambda t_{2}}\right)^{Q \lambda^{\prime} / \lambda} e^{-\delta k^{2}\left(t_{2}-t_{1}\right)}
\end{aligned}
$$

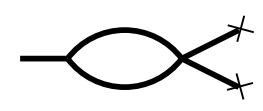

(a)

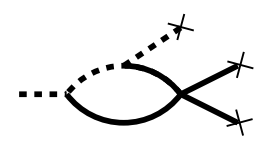

(c)

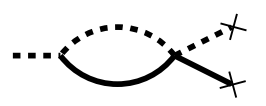

(b)

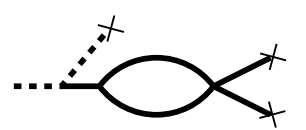

(d)
FIG. 3: (a) One loop contributions to $\langle a(t)\rangle$ and (b)-(d) one loop contributions to $\langle b(t)\rangle$, constructed from the tree level densities and dressed propagators.

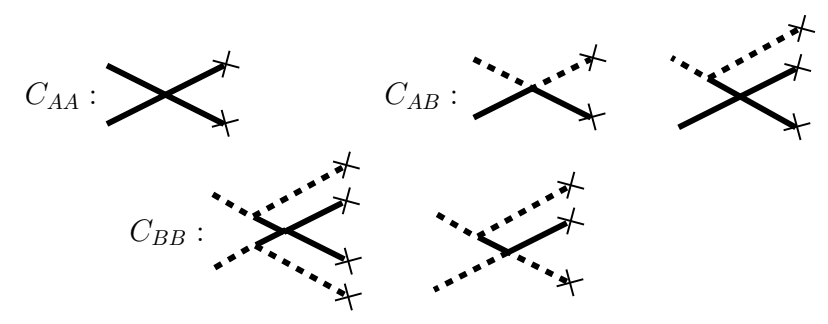

FIG. 4: Tree level correlation functions.

with $G_{\mathrm{tr}}^{A A}=G_{\mathrm{tr}}^{B B}=0$ for $t_{2}<t_{1}$. Notice that the initial density contributions break time translation invariance in the dressed propagators.

With these tree level quantities we can calculate, for example, all one-loop diagrams contributing to $\langle a(t)\rangle$ and $\langle b(t)\rangle$. These require one terminal $a$ or $b$ propagator at time $t$, as shown in the Feynman diagrams of Fig. 3, Additionally, the tree level diagrams for the (unscaled) correlation functions $C_{A A}(r, t)=\langle a(r, t) a(0, t)\rangle-\langle a(t)\rangle^{2}$ and similarly defined $C_{A B}$ and $C_{B B}$ are shown in Fig. 4.

Note the similar topology of the diagrams. The $\langle b(t)\rangle$ diagrams in Fig. 3 can be constructed from diagram (a) by inserting a dashed $B$ line either before the loop, inside the loop, or after the loop, resulting in diagrams (b), (c), and (d) respectively. Similarly, the $C_{A B}$ and $C_{B B}$ correlations are constructed by adding $B$ lines to the $C_{A A}$ diagram. This technique proves useful in generating the one-loop contributions to $C_{B B}$.

\section{RENORMALIZATION}

The bare diagrammatic loop expansion fails to converge for large $t$ when $d \leq 2$, indicating the necessity of renormalizing the theory. Following standard procedure [5] we introduce a normalization time $t_{0}$ and define the dimensionless coupling constants $g_{0}=\lambda t_{0}^{\epsilon / 2}$ and $g_{0}^{\prime}=\lambda^{\prime} t_{0}^{\epsilon / 2}$. The renormalization of the vertices takes the unusually simple form of a geometric sum [3, 14, 23], giving

$$
g_{R}=\frac{g_{0}}{1+g_{0} / g_{*}}, \quad g_{R}^{\prime}=\frac{g_{0}^{\prime}}{1+g_{0}^{\prime} / g_{*}^{\prime}},
$$


with

$$
\begin{aligned}
& g_{*}=\frac{(8 \pi)^{d / 2}}{2 \Gamma(\epsilon / 2)}=2 \pi \epsilon+O\left(\epsilon^{2}\right) \\
& g_{*}^{\prime}=\frac{[4 \pi(1+\delta)]^{d / 2}}{\Gamma(\epsilon / 2)}=(1+\delta) 2 \pi \epsilon+O\left(\epsilon^{2}\right) .
\end{aligned}
$$

For the two-species reaction of Eq. (11), additional field renormalization is required for $B$ particles: the density $b_{B}$ calculated from the bare theory is related to the renormalized density $b_{R}$ via $b_{B}=Z_{b} b_{R}$, with $Z_{b}\left(g_{R}, g_{R}^{\prime}\right)$ chosen to ensure that the expansion of $b_{R}$ in powers of $g_{R}$ has nonsingular coefficients in $\epsilon$. From dimensional analysis and the fact that $b_{B}$ does not depend on the normalization time $t_{0}$ we obtain the RG equation

$$
\begin{array}{r}
{\left[t \frac{\partial}{\partial t}-\frac{d}{2} a_{0} \frac{\partial}{\partial a_{0}}+\frac{1}{2} \beta\left(g_{R}\right) \frac{\partial}{\partial g_{R}}+\frac{1}{2} \beta\left(g_{R}^{\prime}\right) \frac{\partial}{\partial g_{R}^{\prime}}\right.} \\
\left.+\frac{1}{2} \gamma_{b}\left(g_{R}, g_{R}^{\prime}\right)\right] b_{R}\left(t, a_{0}, g_{R}, g_{R}^{\prime} ; t_{0}\right)=0
\end{array}
$$

where

$$
\begin{aligned}
& \beta\left(g_{R}\right) \equiv-2 t_{0} \frac{\partial}{\partial t_{0}} g_{R}=-\epsilon g_{R}+\frac{\epsilon}{g_{*}} g_{R}^{2}, \\
& \beta\left(g_{R}^{\prime}\right) \equiv-2 t_{0} \frac{\partial}{\partial t_{0}} g_{R}^{\prime}=-\epsilon g_{R}^{\prime}+\frac{\epsilon}{g_{*}^{\prime}} g_{R}^{\prime 2},
\end{aligned}
$$

and

$$
\gamma_{b}\left(g_{R}, g_{R}^{\prime}\right) \equiv-2 t_{0} \frac{\partial}{\partial t_{0}} \ln Z_{b}
$$

with $Z_{b}$ yet to be determined. Eq. (20) is solved by the method of characteristics, leading to the asymptotic solution

$$
\begin{aligned}
& b_{R}\left(t, a_{0}, g_{R}, g_{R}^{\prime} ; t_{0}\right) \\
& \quad \sim\left(t / t_{0}\right)^{-\gamma_{b}^{*} / 2} b_{R}\left(t_{0}, a_{0}\left(t / t_{0}\right)^{d / 2}, g_{*}, g_{*}^{\prime} ; t_{0}\right),
\end{aligned}
$$

where $\gamma_{b}^{*}=\gamma_{b}\left(g_{*}, g_{*}^{\prime}\right)$. The general strategy is to compute the bare Feynman diagrams and express the couplings in terms of $g_{R}$ and $g_{R}^{\prime}$, which then flow to their fixed points $g_{*}$ and $g_{*}^{\prime}$ on the right hand side of Eq. (24). Then the asymptotic time dependence of $b_{R}$ is determined by the renormalized $a_{0}$ and the anomalous dimen$\operatorname{sion} \gamma_{b}^{*}$.

The bare tree level and one loop diagrams for the $B$ density have been evaluated in Refs. [14, 16] and can be written as

$$
b_{B}=\frac{b_{0}}{\left(1+a_{0} \lambda t\right)^{Q \lambda^{\prime} / \lambda}}\left[1+\lambda t^{\epsilon / 2}\left(\frac{A(z)}{\epsilon^{2}}+\frac{B(z)}{\epsilon}+\ldots\right)\right]
$$

with the coupling constant ratio

$$
z \equiv \frac{\lambda^{\prime}}{\lambda}=\frac{g_{0}^{\prime}}{g_{0}}
$$

The ratio of bare coupling constants can be expressed in terms of the renormalized couplings as

$$
z=\frac{g_{R}^{\prime}}{g_{R}}\left[1+\sum_{k=0}^{\infty}\left(\frac{g_{R}^{\prime}}{g_{*}^{\prime}}-\frac{g_{R}}{g_{*}}\right) \frac{g_{R}^{\prime k}}{g_{*}^{\prime k}}\right] .
$$

As $g_{R}$ and $g_{R}^{\prime}$ flow to their fixed point values, all terms in the expansion cancel and so $z$ flows to the fixed point value

$$
z^{*}=\frac{g_{*}^{\prime}}{g_{*}}=2\left(\frac{1+\delta}{2}\right)^{d / 2}=1+\delta+O(\epsilon) .
$$

Since $A(z)=-Q z(8 \pi)^{\epsilon / 2} \pi^{-1}\left(1-z / z^{*}\right)$ vanishes as $z \rightarrow z^{*}$, the $A(z) / \epsilon^{2}$ contribution under renormalization is subleading in time and can be neglected. Similarly, $B(z)$ can be expanded in powers of $z-z^{*}$ with only the leading

$$
B\left(z^{*}\right)=\frac{1}{4 \pi}\left(3 Q(1+\delta)+Q^{2}(1+\delta)^{2} f(\delta)\right)+O(\epsilon)
$$

contributing to the asymptotic time dependence, where

$f(\delta)=1+2 \delta\left[\ln \left(\frac{2}{1+\delta}\right)-1\right]+\left(1-\delta^{2}\right)\left[\operatorname{Li}_{2}\left(\frac{\delta-1}{\delta+1}\right)-\frac{\pi^{2}}{6}\right]$

and $\operatorname{Li}_{2}(v)=-\int_{0}^{v} d u \ln (1-u) / u$ is the dilogarithm function [24]. A useful special case is $f(1)=-1$.

Substituting $t \rightarrow t_{0}, a_{0} \rightarrow a_{0}\left(t / t_{0}\right)^{d / 2}$, and $\lambda \rightarrow$ $t_{0}^{-\epsilon / 2} g_{0}=t_{0}^{-\epsilon / 2}\left(g_{R}+g_{R}^{2} / g_{*}+\ldots\right)$ into (25) and then expanding in powers of $g_{R}$ and $g_{R}^{\prime}$ gives to linear order

$$
b_{B}=\frac{b_{0}}{\left(a_{0} g_{R} t^{d / 2}\right)^{Q g_{R}^{\prime} / g_{R}}}\left[1+\frac{B\left(z^{*}\right)}{\epsilon} g_{R}-\frac{Q}{g_{*}} g_{R}^{\prime}+\ldots\right] \text {. }
$$

The $1 / \epsilon$ coefficient in the $g_{R}$ expansion is evidence that field renormalization is required, and allows us to identify $Z_{b}$ to linear order in the couplings and leading order in $\epsilon$ as

$$
Z_{b}=1+\frac{B\left(z^{*}\right)}{\epsilon} g_{R}-\frac{Q}{g_{*}} g_{R}^{\prime}+\ldots
$$

which in turn gives

$$
\gamma_{b}=-B\left(z^{*}\right) g_{R}+\frac{Q}{2 \pi} g_{R}^{\prime} .
$$

Evaluating this at the fixed point couplings and recalling that $Q=1 /(2-p)$ gives

$$
\gamma_{b}^{*}=-\left[\frac{1}{2}\left(\frac{1+\delta}{2-p}\right)+\frac{1}{2}\left(\frac{1+\delta}{2-p}\right)^{2} f(\delta)\right] \epsilon+O\left(\epsilon^{2}\right),
$$

with $f(\delta)$ from Eq. (30). Finally, the renormalized asymptotic density $b_{R}$ then has via Eq. (24) the asymptotic time dependence

$$
b_{R}=Z_{b}^{-1} b_{B} \sim t^{-d Q z^{*} / 2-\gamma_{b}^{*} / 2}=t^{-\theta} .
$$


The renormalized tree level contribution $d Q z^{*} / 2$ is exactly the Smoluchowski exponent, thus we obtain Eq. (4) as claimed.

Our result (4) matches two previous RG calculations which utilized slightly different techniques: in Ref. [14] the $\epsilon \rightarrow 0$ and large $t$ limit were swapped and an expansion in $\ln t$ was resummed, and in Ref. [16] the logarithmic derivative of the density was renormalized instead of the density. The field renormalization approach presented here was previously employed for the special case of $\delta=1$ [15], which we have extended to general $\delta$. As we shall show in the next section, this method is useful for determining the correlation function anomalous dimension $\phi$.

\section{IV. $\phi$ CALCULATION}

An additional independent dynamical exponent appears in the $B$ particle correlation function, resulting from the fact that the $b^{2}$ field requires a distinct renormalization constant $Z_{b^{2}}$ that is not equivalent to $\left(Z_{b}\right)^{2}$. We demonstrate this by calculating the (unscaled) correlation function $C_{B B}(\mathbf{r}, t)=\langle b(\mathbf{r}, t) b(0, t)\rangle-\langle b(t)\rangle^{2}$. For simplicity, we work with the Fourier transform

$$
\hat{C}_{B B}(\mathbf{k}, t)=\int C_{B B}(\mathbf{r}, t) e^{i \mathbf{k} \cdot \mathbf{r}} d^{d} x
$$

at $\mathbf{k}=0$. Assuming the scaling form (5), we expect $\hat{C}_{B B}(k=0, t) \sim t^{\phi-2 \theta+d / 2}$. We introduce the renormalization constant $Z_{b^{2}}$ which relates the bare correlation function to the renormalized one, $\hat{C}_{B B}^{B}=Z_{b^{2}} \hat{C}_{B B}^{R}$, and obtain a similar RG equation

$$
\begin{aligned}
{\left[t \frac{\partial}{\partial t}-\right.} & \frac{d}{2} a_{0} \frac{\partial}{\partial a_{0}}+\frac{1}{2} \beta\left(g_{R}\right) \frac{\partial}{\partial g_{R}}+\frac{1}{2} \beta\left(g_{R}^{\prime}\right) \frac{\partial}{\partial g_{R}^{\prime}} \\
& \left.+\frac{1}{2} \gamma_{b^{2}}\left(g_{R}, g_{R}^{\prime}\right)-\frac{d}{2}\right] \hat{C}_{B B}^{R}\left(t, a_{0}, g_{R}, g_{R}^{\prime} ; t_{0}\right)=0
\end{aligned}
$$

with

$$
\gamma_{b^{2}}\left(g_{R}, g_{R}^{\prime}\right) \equiv-2 t_{0} \frac{\partial}{\partial t_{0}} \ln Z_{b^{2}}
$$

yet to be determined. The method of characteristics solution is

$$
\begin{aligned}
\hat{C}_{B B}^{R}\left(t, a_{0}, g_{R}, g_{R}^{\prime} ; t_{0}\right) & \\
& \sim\left(\frac{t}{t_{0}}\right)^{d / 2-\gamma_{b^{2}}^{*} / 2} \hat{C}_{B B}^{R}\left(t_{0}, a_{0}\left(t / t_{0}\right)^{d / 2}, g_{*}, g_{*}^{\prime} ; t_{0}\right) .
\end{aligned}
$$

The tree-level diagrams in Fig. 4 and the one loop diagrams in the appendix give the bare result

$\hat{C}_{B B}^{B}=\frac{b_{0}^{2} t \lambda^{\prime 2} h(Q)}{\lambda\left(a_{0} \lambda t\right)^{2 Q \lambda^{\prime} / \lambda}}\left[1+\lambda t^{\epsilon / 2}\left(\frac{C(z)}{\epsilon^{2}}+\frac{D(z)}{\epsilon}+\ldots\right)\right]$, where we have taken the large $a_{0}$ limit in anticipation of the RG flow, and $h(Q)=Q(1-2 Q / 3)$. The calculation of the one-loop terms involves 61 distinct Feynman diagrams, the details of which are presented in the appendix. From Eqs. (A3) and (A5) we find, as with the density calculation, that $C(z) \propto z-z *$, making this term subleading in time. From Eqs. (A2), (A3), (A4), and (A5) we obtain

$$
\begin{aligned}
D\left(z^{*}\right)= & -\frac{9-19 Q-27 Q(1+\delta)+18 Q^{2}(1+\delta)}{6 \pi(3-2 Q)} \\
& +\frac{Q^{2}(1+\delta)^{2} f(\delta)}{2 \pi}+O(\epsilon)
\end{aligned}
$$

The procedure described after Eq. (31) provides $\hat{C}_{B B}^{B}$ as an expansion in the renormalized couplings

$$
\begin{aligned}
\hat{C}_{B B}^{B}=\frac{b_{0}^{2} g_{R}^{\prime 2} h(Q)}{g_{R}\left(a_{0} g_{R} t^{d / 2}\right)^{2 Q g_{R}^{\prime} / g_{R}}} & {\left[1+\left(\frac{D\left(z^{*}\right)}{\epsilon}-\frac{1}{g_{*}}\right) g_{R}\right.} \\
& \left.+\left(\frac{2}{g_{*}^{\prime}}-\frac{2 Q}{g_{*}}\right) g_{R}^{\prime}+\ldots\right]
\end{aligned}
$$

The singular coefficients of the $g_{R}$ and $g_{R}^{\prime}$ expansions again indicates the need for field renormalization, so we identify to linear order in the couplings and to leading order in $\epsilon$

$$
Z_{b^{2}}=1+\left(\frac{D\left(z^{*}\right)}{\epsilon}-\frac{1}{g_{*}}\right) g_{R}+\left(\frac{2}{g_{*}^{\prime}}-\frac{2 Q}{g_{*}}\right) g_{R}^{\prime}
$$

which gives

$$
\gamma_{b^{2}}=\left(-D\left(z^{*}\right)+\frac{1}{2 \pi}\right) g_{R}+\left(\frac{Q}{\pi}-\frac{1}{\pi(1+\delta)}\right) g_{R}^{\prime}
$$

This evaluates at the fixed point couplings to

$$
\gamma_{b^{2}}^{*}=-\left[\frac{13}{12-9 p}+\frac{1+\delta}{2-p}+\left(\frac{1+\delta}{2-p}\right)^{2} f(\delta)\right] \epsilon+O\left(\epsilon^{2}\right)
$$

We obtain from (39) the asymptotic form of the renormalized correlation function

$$
\hat{C}_{B B}^{R}(k=0) \sim t^{d / 2-\gamma_{b^{2}}^{*} / 2-d Q z^{*}}
$$

Comparison with the expected time dependence of $t^{d / 2-2 \theta+\phi}$ results in

$$
\phi=\gamma_{b}^{*}-\frac{1}{2} \gamma_{b^{2}}^{*}
$$

which evaluates to the expression given in Eq. (6).

\section{LOGARITHMIC CORRECTIONS IN $d=2$}

At the upper critical dimension of $d=2$ the $\beta$ functions for the renormalized couplings become

$$
\beta\left(g_{R}\right)=\frac{g_{R}^{2}}{2 \pi} \quad \beta\left(g_{R}^{\prime}\right)=\frac{g_{R}^{\prime 2}}{2 \pi(1+\delta)} .
$$


These result in the asymptotic running couplings going to zero as $\tilde{g}_{R} \sim 4 \pi / \ln t$ and $\tilde{g}_{R}^{\prime} \sim 4 \pi(1+\delta) / \ln t$, typical for marginal operators. Plugging these into the $\gamma$-functions gives

$$
\gamma_{b}\left(\tilde{g}_{R}, \tilde{g}_{R}^{\prime}\right) \sim 2 \Gamma_{b} / \ln t \quad \gamma_{b^{2}}\left(\tilde{g}_{R}, \tilde{g}_{R}^{\prime}\right) \sim 2 \Gamma_{b^{2}} / \ln t
$$

where

$$
\Gamma_{b}=\lim _{\epsilon \rightarrow 0} \frac{\gamma_{b}^{*}}{\epsilon} \quad \Gamma_{b^{2}}=\lim _{\epsilon \rightarrow 0} \frac{\gamma_{b^{2}}^{*}}{\epsilon}
$$

Inserting this into the density RG equation (20) gives the asymptotic solution

$$
\begin{aligned}
& b_{R}\left(t, a_{0}, g_{R}, g_{R}^{\prime} ; t_{0}\right)= \\
& \quad \sim \ln \left(t / t_{0}\right)^{-\Gamma_{b}} b_{R}\left(t_{0}, a_{0} t / t_{0}, \tilde{g}_{R}(t), \tilde{g}_{R}^{\prime}(t) ; t_{0}\right)
\end{aligned}
$$

which results in the density given in Eqs. (7) and (2). As a check on this result, for $p=0$ ( $A$ particle annihilation) and $\delta=1$ the $B$ particle density should match that of the $A$ particles, which is known to decay as $\ln t / t$ in $d=2$ [2, 3]. Our expression is consistent with this.

Inserting the running couplings into the RG equation (37) for the (unscaled) correlations $\hat{C}_{B B}(k=0)$ gives the method of characteristics solution

$$
\begin{aligned}
& \hat{C}_{B B}^{R}\left(t, a_{0}, g_{R}, g_{R}^{\prime} ; t_{0}\right) \\
& \quad \sim\left(\frac{t}{t_{0}}\right) \ln \left(t / t_{0}\right)^{-\Gamma_{b^{2}}} \hat{C}_{B B}^{R}\left(t_{0}, a_{0} t / t_{0}, \tilde{g}_{R}(t), \tilde{g}_{R}^{\prime}(t) ; t_{0}\right) .
\end{aligned}
$$

This results in the asymptotic time dependence

$$
\hat{C}_{B B}^{R}(k=0) \sim t^{1-2(1+\delta) /(2-p)}(\ln t)^{-\Gamma_{b^{2}}-1+2(1+\delta) /(2-p)} .
$$

Transforming back to real space and dividing by the density squared results in $\beta=2 \Gamma_{b}-\Gamma_{b^{2}}-1$ and the correlation function scaling given in Eqs. (9) and (10).

\section{NUMERICAL SOLUTION FOR $A+A \rightarrow A$ IN $d=1$}

In one spatial dimension and for the case $p=1(A$ particle coalescence) it is known that the density decay exponent $\theta$ can be determined from the problem of three vicious walkers [25]. For any $B$ particle there are nearest neighbor $A$ particles to the left and right which undergo simple random walks, since the product of any future coalescence events can be identified as the original $A$ neighbor. Appealing to the universality of $\theta$, we may consider the limit of an infinite reaction rate for $A+B \rightarrow A$, which implies the $B$ particle density decay is equivalent to the survival probability of the three vicious walker problem [26], giving

$$
\theta=\frac{\pi}{2 \arccos (\delta /(1+\delta))}
$$

In a similar way, the anomalous dimension $\phi$ of the $C_{B B}$ correlation function is related to a four walker problem in which middle two walkers ( $B$ 's) are allowed to meet any number of times, but have had no encounters with the leftmost and rightmost walkers $(A$ 's), i.e., the walkers' positions obey $x_{1}<x_{2}, x_{3}<x_{4}$. We refer to this as the bracket problem. Further, the power-law decay of $\left\langle b^{2}\right\rangle \sim t^{-2 \theta+\phi}$ is given by the probability that not only have both middle walkers survived, but they have also reached the same location $\left(x_{2}=x_{3}\right)$ at time $t$. This exponent cannot be simply determined analytically, but it is possible to map the calculation to an electrostatic problem, which allows for an accurate numerical determination. In what follows we limit consideration to the equal diffusion constant case, $\delta=1$.

Since the center of mass motion plays no role in the absorption probability, the coordinates $x_{i}$ of the four walkers can be projected to the three dimensional subspace $x_{1}+x_{2}+x_{3}+x_{4}=0$ and parametrized in terms of the coordinates $u_{i}=\left(x_{i}+x_{4}\right) / 2$ for $i=1,2,3$. The four walker dynamics maps to isotropic diffusion in this three dimensional space, the geometry of which is clearly mapped out in Ref. [19]: the six planes $u_{k}= \pm u_{\ell}, k \neq \ell$ correspond to the six possible particle encounters $x_{i}=x_{j}, i \neq j$ and divide space into 24 wedges, each corresponding to a permutation of the ordering of the four walkers. The four vicious walker problem reduces to the survival probability of a diffusing particle in the wedge $\left|u_{1}\right|<u_{2}<u_{3}$ with absorbing boundary conditions at $u_{1}= \pm u_{2}$ and $u_{2}=u_{3}$. The bracket problem corresponds instead to a wedge $\left|u_{1}\right|<u_{2}$ and $\left|u_{1}\right|<u_{3}$ with absorbing boundary conditions at $u_{1}= \pm u_{2}$ and $u_{1}= \pm u_{3}$. By symmetry the bracket problem wedge is equivalent to the smaller vicious walker wedge with a reflective boundary at $u_{2}=u_{3}$.

The time-dependent probability density $p(\mathbf{r}, t)$ of a walker that starts at $\mathbf{r}_{0}$ obeys the diffusion equation with initial condition $p(\mathbf{r}, 0)=\delta\left(\mathbf{r}-\mathbf{r}_{0}\right)$ and $p\left(\mathbf{r}^{\prime}, t\right)=0$ for $\mathbf{r}^{\prime}$ on the boundary. Because of the absorbing boundaries, the probability density is not normalized for $t>0$. For such scale-free wedges the asymptotic behavior for $r \gg r_{0}$ and $\sqrt{D t} \gg r_{0}$ is given by

$$
p(\mathbf{r}, t)=C r^{\eta} t^{-\eta-d / 2} e^{-r^{2} / 4 D t} f(\hat{\mathbf{r}})
$$

where the exponent $\eta$ is related to the smallest eigenvalue of the spherical laplacian $\nabla_{S_{d}}^{2}$ acting in the wedge geometry with Dirichlet boundary conditions, and $f(\hat{\mathbf{r}})$ is the corresponding eigenfunction [27, 28]. The constant $C$ depends on the initial location of the particle.

In simple geometries, such as a cone, this eigenvalue problem can be solved analytically [29] to obtain the value of $\eta$, but this is difficult for most wedges. Instead we exploit the fact that

$$
V(\mathbf{r}) \equiv \int_{0}^{\infty} p(\mathbf{r}, t) d t
$$

obeys Poisson's equation with source $(1 / D) \delta\left(\mathbf{r}-\mathbf{r}_{0}\right)[18]$. We can solve this electrostatic problem numerically to 
find the large $r$ behavior $V \sim r^{-\mu}$, and then (56) implies $\eta=\mu-d+2$.

Finally, the survival probability of the bracket problem is given by $S(t)=\int p(\mathbf{r}, t) d^{d} r \sim t^{-\beta}$, where the integral is over the wedge volume. From (55) it follows that $\beta=$ $\eta / 2$. However, for our exponent $\phi$ we need to impose the additional constraint that the two center walkers meet at time $t$, which reduces the dimension of the spatial integral by one, i.e.,

$$
\left\langle b^{2}(t)\right\rangle \sim \bar{S}(t) \sim \int p(\mathbf{r}, t) d^{d-1} r \sim t^{-\bar{\beta}},
$$

giving $\bar{\beta}=(\eta+1) / 2$. Using the known value of $\theta=3 / 2$ for this equal diffusion constant case, we get $\phi=3-\bar{\beta}=$ $3-\mu / 2$.

We solved Poisson's equation for the bracket problem by successive over relaxation on an integer lattice with $u_{i}^{\max }=500$. A point charge was located at $\left(u_{1}, u_{2}, u_{3}\right)=(0,1,1)$, with absorbing boundary conditions at $u_{1}= \pm u_{2}$ and reflecting boundary conditions at $u_{2}=u_{3}$. Following [19], we did two separate calculations with the boundary conditions at the edge of the box, $u_{3}=u_{3}^{\max }$, taken to be either absorbing $(V=0)$ or reflecting $\left(\partial V / \partial u_{3}=0\right)$. The resulting solutions bound the infinite wedge solution. We fit the data to the form $V \sim A r^{-\mu}\left(1+B r^{-2}\right)$ to account for the discreteness of the lattice laplacian and estimate the uncertainty by fitting $V(r)$ along seven different directions: $(0,1,1)$, $(0,1,2),(1,2,2),(0,1,3),(0,2,3),(1,2,3)$, and $(0,1,4)$. We obtain $\mu=4.747507(6)$, which implies $\eta=3.747507(6)$ in Eq. (55) and the values for $\beta$ and $\phi$ reported in the introduction.

\section{SUMMARY AND FUTURE WORK}

We have shown that the two-species reaction diffusion system described by Eq. (10) exhibits anomalous dimension, not only in the $B$ particle density but also in the $B B$ correlation function. We demonstrated the universality of the anomalous scaling of the correlation function, Eq. (5), and computed the exponent $\phi$ to first order in $\epsilon$. Surprisingly the first order term exhibits no dependence on the diffusion constant ratio $\delta$. The exponent $\phi$ goes to zero as $d \rightarrow 2$ from below, continuously connecting to the $d>2$ value, in contrast to the density decay exponent.

At the critical dimension $d=2$ we have shown that both the $B$ particle density and the scaled $\tilde{C}_{B B}$ correlation function acquire logarithmic corrections, and have computed the associated exponents. Our results match previous calculations of the density decay exponent, [14, 16], though differing on the exponent $\alpha$ for the logarithmic corrections. As previously noted [16], Ref. [14] did not fully incorporate loop corrections. Our discrepancy with Ref. [16] is more troublesome. They found for $\delta \neq 1$ an additional nonuniversal contribution to the logarithm exponent that does not arise in our approach, and supported their calculation with numerical evidence for $\delta=0$. Their technique was to renormalize the logarthmic derivative of the bare density $t \partial_{t} \ln \langle b(t)\rangle$, rather than renormalizing the density itself. It is possible that these two approaches, which both involve a concurrent $\tilde{a}_{0} \rightarrow \infty$ limit, are not equivalent. This issue merits future study.

We derived the anomalous dimension by renormalizing the $b$ and $b^{2}$ fields by the factors $Z_{b}$ and $Z_{b^{2}}$. Since the density is exactly proportional to the initial density $b_{0}$ and the correlation function is exactly proportional to $b_{0}^{2}$, one may equivalently view the constants $Z_{b}$ and $Z_{b^{2}}$ as renormalizing $b_{0}$ and $b_{0}^{2}$. This has an appealing physical interpretation: as the $A$ particle correlations approach their universal scaling form, the effective reaction rate is renormalized, requiring an adjustment in the initial density of $B$ particles to compensate. That $b_{0}^{2}$ requires a distinct renormalization indicates that the fluctuations in the $B$ particles must be modified as well. An interesting direction for future work would be to explore what minimum ingredients in a reaction-diffusion system are sufficient to require field renormalization.

For the special case of $p=1$ in dimension $d=1$ we have determined $\phi$ from the four walker bracket problem. This approach strongly suggests that the exponent should depend on $\delta$, since varying the parameter from unity has the effect of opening or closing the angle of the absorbing wedge in the four walker problem, which should modify the survival probability decay exponent. This could be investigated numerically.

We are currently undertaking a simulation of this reaction-diffusion system that utilizes a variation of the approach of Mehra and Grassberger [30]. These authors studied the trapping reaction, $A+B \rightarrow B$, and developed a method for tracking the entire $A$ particle distribution conditioned on a realized trajectory of a single $B$ particle. For our system, Eq. (11), this can be inverted: the $A$ particle dynamics can be treated via Monte Carlo, and for a given realization of the $A$ particles, the entire $B$ particle distribution can be generated. This method should allow for reasonably high quality statistics to test the predicted anomalous scaling for the $C_{B B}$ correlation function, and to explore the dependence of the dynamical exponents $\theta$ and $\phi$ on the parameters $p$ and $\delta$.

Finally, the lack of rapid convergence of the $\epsilon$ expansion appears to be a general feature of these RG fixed points, also observed in the single-species annihilation reaction [4]. For that system, Vernon showed that replacing the short-range diffusive hops with Lévy flights, governed by a size distribution $P(r) \sim r^{-d-\sigma}$ with $1<\sigma<2$, lowered the upper critical dimension to $d_{c}=\sigma$ [31]. Thus $\sigma$ can be chosen so that $\epsilon=\sigma-d$ is small in $d=1$ simulations, which allowed Vernon to confirm the accuracy of the RG $\epsilon$ expansion [31]. Such an approach could be interesting here, in particular to test whether the $\delta$ dependence of $\phi$ weakens as $\epsilon$ becomes small. 

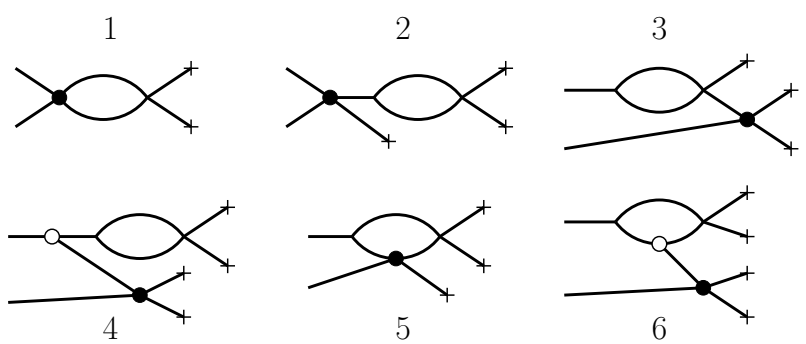

FIG. 5: The six topology classes of diagrams (not yet including the dashed $B$ particle lines). The solid circle identifies $\lambda_{4}$ and the open circle $\lambda_{3}$. Description: (1) $\lambda_{4}$ closes loop, (2) loop in causal past of $\lambda_{4}$, (3) $\lambda_{4}$ in causal past of loop, (4) loop and $\lambda_{4}$ in causal past of $\lambda_{3}$, (5) $\lambda_{4}$ in loop, and (6) $\lambda_{3}$ in loop.

\section{Acknowledgments}

J.H. and R.S.M. were supported by NSF REU Grant PHY-1156964 and J.H. was supported by NSF Grant DMS-1612921. B.P.V.-L. acknowledges the hospitality of the University of Göttingen, where this work was completed.

\section{Appendix A: One Loop Correlation Diagrams}

Many diagrams contribute to $\hat{C}_{B B}(k=0)$ at one loop, and care must be taken to identify them. All have exactly two four-point vertices: one that begins the loop and one that links the two terminal lines. The latter vertex we label $\lambda_{4}$. Some diagrams have a particular three point vertex, which we label $\lambda_{3}$, that connects $\lambda_{4}$ to the loop. All possible diagrams fall into six topology classes, as shown in Fig. 5]. Adding the dashed $B$ lines to these in every distinct way results in a total of 61 diagrams. We present our results in the form

$$
\hat{C}_{B B}^{1 \text {-loop }}(k=0)=\frac{b_{0}^{2} t^{1+\epsilon / 2}}{\left(a_{0} \lambda t\right)^{2 Q \lambda^{\prime} / \lambda}} \frac{Q \lambda^{\prime 2}}{12 \pi} \sum_{i=1}^{6} F_{i}(z, \epsilon)
$$

where the $F_{i}$ are the contributions from each class of diagram, and $z=\lambda^{\prime} / \lambda$.

A remark on the order of $\epsilon$ : some diagrams contain order $1 / \epsilon^{2}$ contributions. As with the density calculation, we will show that these terms cancel as the couplings flow to their fixed point values, and $z \rightarrow z^{*}$. The $1 / \epsilon$ portions of the diagrams will contribute to the renormalization factor $Z_{b^{2}}$ and ultimately provide the $O(\epsilon)$ contribution to the anomalous dimension. Any diagrams that are finite as $\epsilon \rightarrow 0$ do not contribute to the anomalous dimension and may be neglected.
Class 1:

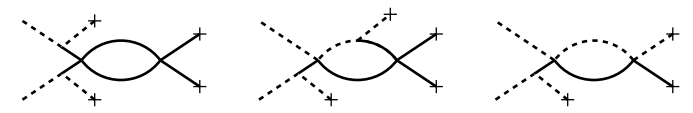

Class 2:

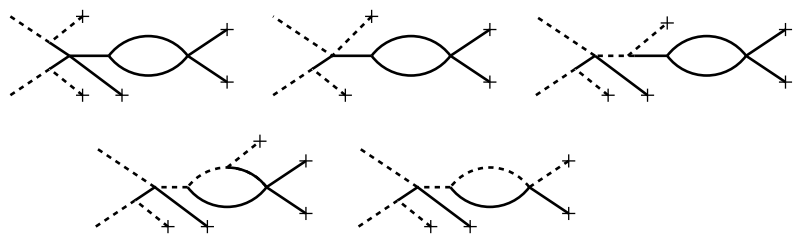

Class 3:
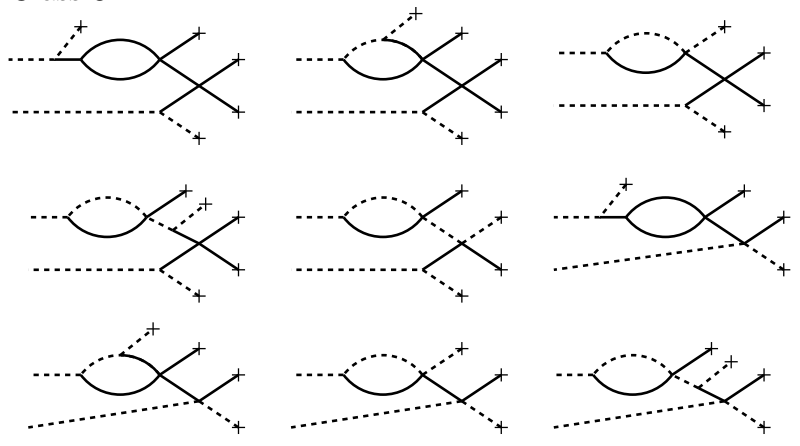

FIG. 6: All one-loop diagrams contributing to $\hat{C}_{B B}(k=0)$ in Classes 1, 2, and 3 .

From the class 1, 2, and 3 diagrams in Fig. 6] we obtain

$$
\begin{aligned}
F_{1} & =\left(4 Q-\frac{6 z}{1+\delta}\right) \frac{1}{\epsilon} \\
F_{2} & =12 Q z(8 \pi)^{\epsilon / 2}\left(1-\frac{z}{z^{*}}\right) \frac{1}{\epsilon^{2}} \\
& +\left(6+24 Q z-4 Q+3 Q^{2} z^{2} f(\delta)-\frac{15 Q z^{2}}{1+\delta}\right) \frac{1}{\epsilon}, \\
F_{3} & =\left(6-\frac{14 Q}{3}+\frac{8 Q z-6 z-10 Q^{2} z^{2}+15 Q z^{2}}{1+\delta}\right) \frac{1}{\epsilon}
\end{aligned}
$$

From the class 4 diagrams in Fig. 7 we obtain

$$
\begin{aligned}
F_{4}= & \left(\frac{4 Q}{3}-1\right) Q z(8 \pi)^{\epsilon / 2}\left(1-\frac{z}{z^{*}}\right) \frac{1}{\epsilon^{2}} \\
+ & \left(-6+6 Q z+\frac{28 Q}{3}-20 Q^{2} z+\frac{18 Q^{2} z^{2}-12 Q z^{2}}{1+\delta}\right. \\
& \left.+\left(3 Q^{2} z^{2}-4 Q^{3} z^{2}\right) f(\delta)\right) \frac{1}{\epsilon}
\end{aligned}
$$

The 18 diagrams in class 5 and the 15 diagrams in class 6 are all finite as $\epsilon \rightarrow 0$ and do not contribute to the anomalous dimension. We do not depict these diagrams here. It is relatively straightforward to evaluate the order of the diagrams: a factor of $1 / \epsilon$ is obtained from any simple loop that does not have a propagator 



FIG. 7: All one-loop diagrams contributing to $\hat{C}_{B B}(k=0)$ in Class 4. attached to it. No such loops exist in classes 5 and 6 . Some diagrams obtain a factor of $1 / \epsilon$ from having a nonloop time integral of the form $\int t^{-1+\epsilon / 2} d t$. These may be identified by dimensional analysis and do not occur in classes 5 and 6 .
[1] Ovchinnikov A and Zeldovich Y 1978 Chem. Phys. 28215

[2] Toussaint D and Wilczek F 1983 J. Chem. Phys. 782642

[3] Peliti L 1986 J. Phys. A 19 L365

[4] Lee B P 1994 J. Phys. A 272633

[5] Täuber U C, Howard M and Vollmayr-Lee B P $2005 \mathrm{~J}$. Phys. A 38 R79

[6] Smoluchowski M V 1917 Z. Phys. Chem. 92129

[7] Chandrasekhar S 1943 Rev. Mod. Phys. 151

[8] Cardy J and Täuber U C 1996 Phys. Rev. Lett. 774780

[9] Cardy J L and Täuber U C 1998 J.Stat. Phys. 901

[10] Hinrichsen H 2000 Adv. Phys. 49815

[11] Janssen H K and Täuber U C 2005 Ann. Phys. 315147

[12] Täuber U C 2014 Critical Dynamics - A Field Theory Approach to Equilibrium and Non-Equilibrium Scaling Behavior (Cambridge: Cambridge University Press)

[13] Krapivsky P L, Ben-Naim E and Redner S 1994 Phys. Rev. E 502474

[14] Howard M 1996 J. Phys. A 293437

[15] Krishnamurthy S, Rajesh R and Zaboronski O 2003 Phys. Rev. E 68046103

[16] Rajesh R and Zaboronski O 2004 Phys. Rev. E 70036111

[17] Munasinghe R M, Rajesh R and Zaboronski O V 2006 Phys. Rev. E $\mathbf{7 3} 051103$

[18] Redner S and Krapivsky P L 1999 Am. J. Phys. 671277

[19] ben Avraham D, Johnson B M, Monaco C A, Krapivsky P L and Redner S 2003 J. Phys. A 361789

[20] Doi M 1976 J. Phys. A 91465

[21] Grassberger P and Scheunert M 1980 Fortschr. Phys. 28 547

[22] Peliti L 1985 J. Phys. 461469

[23] Lee B P and Cardy J 1995 J. Stat. Phys. 80971

[24] Abramowitz M and Stegun I A 1972 Handbook of Mathematical Functions (New York: Dover)

[25] Redner S 2001 A Guide to First-Passage Processes (Cambridge: Cambridge University Press)

[26] Fisher M E and Gelfand M P 1988 J. Stat. Phys. 53175

[27] Hammer Y and Kantor Y 2014 Phys. Rev. E 89022601
[28] Alfasi N and Kantor Y 2015 Phys. Rev. E 91042126

[29] Ben-Naim E and Krapivsky P L 2010 J. Phys. A 43 495007

[30] Mehra V and Grassberger P 2002 Phys. Rev. E 65050101

[31] Vernon D C 2003 Phys. Rev. E 68041103 


\title{
Corrigendum: Anomalous Dimension in a Two-Species Reaction-Diffusion System
}

\author{
Benjamin Vollmayr-Lee ${ }^{1}$, Jack Hanson ${ }^{2}$, R. Scott McIsaac ${ }^{3}$, and Joshua D. Hellerick ${ }^{1}$ \\ ${ }^{1}$ Department of Physics \&S Astronomy, Bucknell University, Lewisburg, PA 17837, USA \\ ${ }^{2}$ Department of Mathematics, City College of New York, 160 Convent Ave, New York, NY 10031 USA \\ ${ }^{3}$ Calico Life Sciences, South San Francisco, CA 94080 USA
}

\begin{abstract}
We make two corrections to the renormalization group calculation presented in J. Phys. A: Math. Theor. 51, 034002 (2018). First, the field renormalization technique presented is not applicable for the $B$ particle density in $d=2$ because of noncommutitivity of the $\epsilon \rightarrow 0$ and $t \rightarrow \infty$ limits. The $B$ particle density in $d<2$ and the correlation function for $d \leq 2$ are unaffected by this issue. Second, we correct a symmetry factor in one of the diagrams, which modifies the correlation function scaling exponents.
\end{abstract}

The renormalization group calculation presented in 1] contains two errors. The first concerns the $B$ particle density at the upper critical dimension $d=2$, which decays as $\langle b\rangle \sim t^{-\theta}(\ln t)^{\alpha}$. Our calculation of $\alpha$ was based on the assumption that the contribution $A(z) / \epsilon^{2}$ in Eq. (25) was asymptotically negligible because

$$
A(z) \propto\left(z-z^{*}\right) \sim \begin{cases}t^{-\epsilon / 2} & d<2 \\ 1 / \ln t & d=2 .\end{cases}
$$

This assumption is valid for $d<2$ since for any $\epsilon>0$, there exists an asymptotic regime where $1 /\left(\epsilon^{2} t^{\epsilon / 2}\right)$ is negligibly small. But for $d=2$ we must take the $\epsilon \rightarrow 0$ limit before the large $t$ limit, and this term cannot be neglected. As a result, the field renormalization technique employed in [1] is not applicable and one must instead employ the technique of Rajesh and Zaboronski [2], where they renormalize instead the logarithmic derivative $t \partial_{t} \ln \langle b\rangle$. Their result agrees with our Ref. [1] Eq. (7), but their value of $\alpha$, which in our notation reads

$$
\begin{aligned}
\alpha= & \left(\frac{1+\delta}{2-p}\right)\left[\frac{3}{2}+\ln \left(\frac{1+\delta}{2}\right)+\frac{1}{2}\left(\frac{1+\delta}{2-p}\right) f(\delta)\right] \\
& -\frac{4 \pi(1+\delta)}{2-p}\left(\frac{1}{\lambda}-\frac{1+\delta}{\lambda^{\prime}}\right),
\end{aligned}
$$

corrects the value we reported in Eq. (8).

However, the problem of the noncommuting $\epsilon \rightarrow 0$ and $t \rightarrow \infty$ limits does not affect the calculation of correlation function scaling exponents $\phi$ and $\alpha_{2}$, defined via

$$
\tilde{C}_{B B}(r, t)= \begin{cases}t^{\phi} f(r / \sqrt{t}) & d<2 \\ (\ln t)^{\alpha_{2}} f(r / \sqrt{t}) & d=2,\end{cases}
$$

provided one renormalizes the scaled correlation function $\tilde{C}_{B B}(r, t)=C_{B B}(r, t) /\langle b(t)\rangle^{2}$. Our bare expansion for the unscaled $C_{B B}^{B}$ in Eq. (40) is then replaced by

$$
\begin{aligned}
\hat{\tilde{C}}_{B B}^{B}=\frac{t \lambda^{\prime 2} h(Q)}{\lambda}[1 & +\lambda t^{\epsilon / 2}\left(\frac{C(z)-2 A(z)}{\epsilon^{2}}\right. \\
& \left.\left.+\frac{D(z)-2 B(z)}{\epsilon}+\ldots\right)\right] .
\end{aligned}
$$

The $1 / \epsilon^{2}$ term then vanishes because $C(z)=2 A(z)$. The remaining $1 / \epsilon$ term can be controlled by field renormalization as before, with the same final results:

\[ \phi=\left[\pi\left(D\left(z^{*}\right)-2 B(z *)\right)+\frac{1}{2}\right] \epsilon+O\left(\epsilon^{2}\right) \]
for $d<2$ and

$$
\alpha_{2}=2 \pi\left(D\left(z^{*}\right)-2 B\left(z^{*}\right)\right)
$$

for $d=2$.

The second error in [1] was a symmetry factor of two in the first diagram of Class 2 in Fig. A2. The corrected Eq. (A.3) reads

$$
\begin{aligned}
F_{2}= & 12 Q z(8 \pi)^{\epsilon / 2}\left(\frac{z}{z^{*}}-1\right) \frac{1}{\epsilon^{2}} \\
& +\left(6+24 Q z-8 Q+3 Q^{2} z^{2} f(\delta)-\frac{15 Q z^{2}}{1+\delta}\right) \frac{1}{\epsilon}
\end{aligned}
$$

which changes $D\left(z^{*}\right)$ in Eq. (41) to

$D\left(z^{*}\right)=-\frac{9-13 Q}{6 \pi(3-2 Q)}+\frac{3 Q(1+\delta)}{2 \pi}+\frac{Q^{2}(1+\delta)^{2} f(\delta)}{2 \pi}+O(\epsilon)$

This modifies the correlation function exponents: Eq. (6) becomes

$$
\phi=\frac{7}{24-18 p} \epsilon+O\left(\epsilon^{2}\right)
$$

and Eq. (10) becomes

$$
\alpha_{2}=-\frac{5-9 p}{12-9 p}
$$

In the text after Eq. (11), the value of $\phi$ for the truncated $\mathrm{RG}$ expansion in $d=1$ with $p=1$ and $\delta=1$ is $\phi=\frac{7}{6} \simeq$ 1.17 . 
[1] Vollmayr-Lee B, Hanson J, McIsaac R S and Hellerick J D 2018 J. Phys. A: Math. Theor. 51034002

[2] Rajesh R and Zaboronski O 2004 Phys. Rev. E 70036111 